\title{
The potential role of curcumin (diferuloylmethane) in plasma cell dyscrasias/ paraproteinemia
}

\author{
Terry Golombick \\ Terry Diamond \\ Department of Endocrinology, \\ St George Hospital, Kogarah, Australia
}

\begin{abstract}
Plasma cell dyscrasias, most commonly associated with paraproteinemia, are a diverse group of diseases. Monoclonal gammopathy of undefined significance (MGUS) can precede multiple myeloma, a progressive neoplastic disease. MGUS occurs in association with a variety of other diseases and currently no treatment is recommended but rather "watchful waiting". Given that the size of the M-protein is a risk factor for disease progression, early intervention with the aim of reducing the paraprotein load would provide an innovative therapeutic tool. Preliminary results from our pilot study show a drop of between $5 \%$ and $30 \%$ serum paraprotein in patients taking curcumin compared with patients on placebo. Curcumin is a diferuloylmethane present in extracts of the rhizome of the Curcuma longa plant. As a natural product, this has exciting potential in the treatment of plasma cell dyscrasias.
\end{abstract}

Keywords: plasma cell dyscrasias, MGUS, myeloma, curcumin, paraproteinemia

A paraprotein is a monoclonal immunoglobulin or immunoglobulin light chain (Bence Jones protein) present in blood or urine and arising from clonal proliferation of mature B cells, most commonly plasma cells or B-lymphocytes. Alternative terms include monoclonal protein or M-band.

Plasma cell dyscrasias, most commonly associated with paraproteinemia, are a diverse group of diseases which includes multiple myeloma, Waldenstrom's macroglobulinemia, heavy chain disease, monoclonal gammopathy of undefined significance (MGUS), and immunocytic amyloidosis. The incidence of plasma cell dyscrasias is age-related, with $1 \%$ of persons over age 25 and $4 \%$ of those over age 70 . Paraproteinemia is also associated with the non-Hodgkin lymphoma disorders CLL (chronic lymphocytic leukemia) and LPL (lymphoplasmacytic lymphoma).

MGUS or monoclonal gammopathy of undefined significance, can precede multiple myeloma and is typified by a serum M-protein value of $<30 \mathrm{~g} / \mathrm{L}$, fewer than $10 \%$ plasma cells in the bone marrow, no or a small amount of $\mathrm{M}$ protein in the urine, and absence of lytic bone lesions, anemia, hypercalcemia or renal insufficiency related to the plasma-cell proliferative process (Kyle and Rajkumar 2005). MGUS occurs in association with a variety of other diseases and currently no treatment is recommended; rather that patients should be observed for change in clinical and immunochemical status at 4-6 month intervals.

Overall the risk of progression of MGUS to myeloma or related disorder is $1 \%$ per year. Although the prevalence of MGUS increases with advancing age, after adjustment for the level of the M-protein, the annual risk of progression to myeloma or a related cancer is not affected by age or the duration of MGUS. Younger patients are more likely to have progression to cancer during their lifetime because they are at 


\section{CURCUMIN - PARAPROTEIN LEVELS (g/L)}

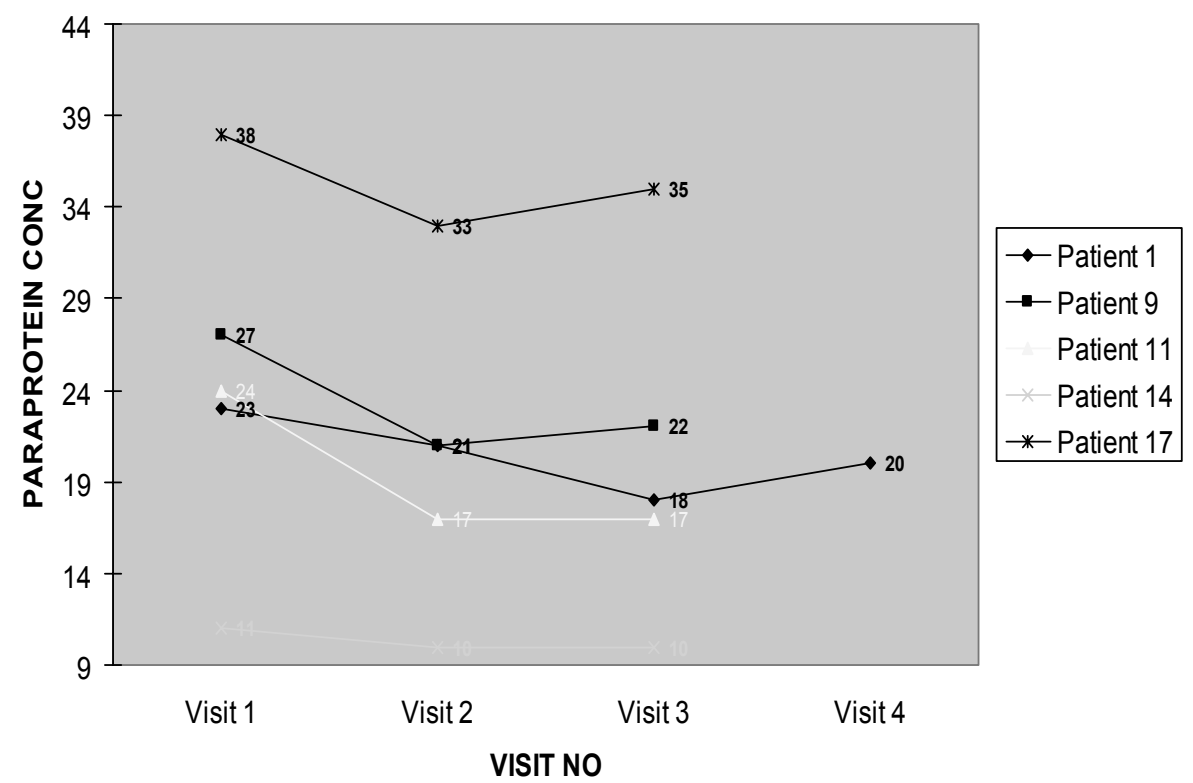

Figure I Curcumin: Paraprotein levels (g/L).

risk for longer (Cook and Macdonald 2007). It is currently not possible to predict the course in any individual patient, and clinically symptomatic myeloma may not evolve for as long as 20 years. Myeloma is a progressive neoplastic disease and is often associated with multiple osteolytic lesions, hypercalcemia, anemia, renal damage, and increased susceptibility to bacterial infections. Myeloma is associated with a high mortality.

Parameters which are currently used to identify those patients at highest risk of developing disease progression

\section{Control - paraprotein levels}

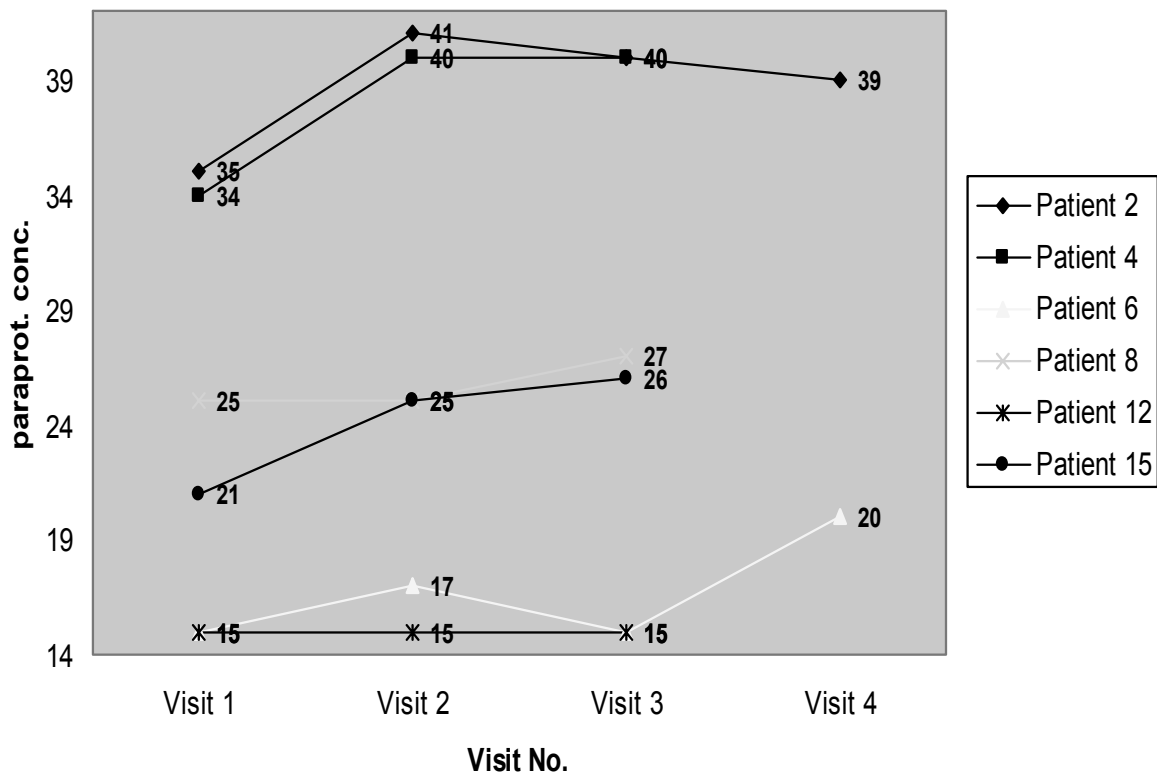

Figure 2 Control: Paraprotein levels. 
are: the size of the M-protein, the type of M-protein with IgA and IgM paraproteins having a higher risk compared with IgG paraproteins, the percentage of bone marrow plasma cells, and an abnormal serum-free light chain ratio. Given the uncertainty of disease progression with MGUS, early intervention with the aim of reducing the paraprotein load would provide an innovative therapeutic tool.

Curcuma longa or turmeric is a tropical plant native to southern and southeastern tropical Asia. It is a perennial herb belonging to the ginger family. The most active component in turmeric is curcumin (Aggarwal et al 2005). Curcumin is a diferuloylmethane present in extracts of the rhizome of the Curcuma longa plant.

This non-nutritive phytochemical is pharmacologically safe, considering that it has been consumed as a dietary spice, at doses up to $100 \mathrm{mg} /$ day, for centuries. Recent phase 1 clinical trials indicate that people can tolerate a dose as high as $8 \mathrm{~g}$ /day with no adverse effects (Sharma et al 2004).

A Medline search revealed over 1500 publications describing various activities of this polyphenol. Curcumin has been shown to suppress proliferation of a wide variety of tumor cells; to down-regulate transcription factors NF-KB, AP-1, and early growth response gene-1; to suppress the expression of cyclooxygenase-2, lipoxygenase, NO synthase, matrix metalloproteinase-9, urokinase-type plasminogen activator, tumor necrosis factor, chemokines, cell surface adhesion molecules and cyclin D1; to inhibit the expression of growth factor receptors (such as epidermal growth factor receptor and human epidermal growth factor receptor 2); and to inhibit the activity of JNK, protein tyrosine kinases, and several other protein serine/threonine kinases. This polyphenol has antioxidant and anti-inflammatory activity and has been found to suppress tumor initiation, promotion and metastasis. Numerous reports suggest that curcumin has chemopreventive and chemotherapeutic effects. Curcumin has been shown to inhibit the proliferation of a wide variety of tumor cells, including multiple myeloma cells through the down-regulation of interleukin-6.

Nine different studies of the safety and efficacy of curcumin in humans have been reported (see Aggarwal et al
2005). Over the past several years, numerous studies have been funded by the National Institutes of Health to investigate the role of curcumin and its derivatives in treatment of patients with cancer. Additionally, M.D. Anderson Cancer Center at the University of Texas is involved in pre-clinical and clinical research of the anti-cancer mechanism and application of curcuminoids in conditions including lung, breast, multiple myeloma, pancreatic, myelodysplatic syndrome, colon, prostate, head, and neck cancers.

How curcumin produces its therapeutic effects is not fully understood. It has been suggested that the effects are in part through the antioxidant and anti-inflammatory action of curcumin. It appears likely that curcumin mediates its effects through other mechanisms as well.

We are conducting a single blind randomized controlled pilot study on 25 patients with paraproteinemia. Entry criteria included patients defined as having MGUS ie, the presence of a serum paraprotein (greater than $8 \mathrm{~g} / \mathrm{L}$ and less than $40 \mathrm{~g} / \mathrm{L}$ ) with the exclusion of multiple myeloma. These patients are being monitored for a 6 month period of curcumin or placebo therapy. Curcumin or placebo is being administered orally as a 2 grams twice daily regimen. After one week on curcumin, there has been a drop of between $5 \%$ and $30 \%$ serum paraprotein in some patients, compared to controls (see figures). After 3 months of curcumin therapy, these reduced levels have remained suppressed. These exciting findings have prompted a double-blind, randomized, controlled trial. The benefits of the fall in paraprotein is uncertain. How long these reduced levels will remain suppressed and what the clinical benefits are, remain to be seen. As a natural product, it has exciting potential in the treatment of plasma cell dyscrasias.

\section{References}

Kyle RA, Rajkumar SV. 2006. Monoclonal gammopathies of undetermined significance. Br J Haematol, 34:573-89.

Cook L, Macdonald DHC. 2007. Management of paraproteinaemia. Postgrad Med J, 83:217-23.

Aggarwal BB, Kumar A, Aggarwal MS, et al. 2005. Curcumin Derived from Turmeric Curcuma longa: a spice for All Seasons. In: Bagchi D (ed). Phytopharmaceuticals in Cancer Chemoprevention, pp. 349-87.

Sharma RA, Euden SA, Platton SL, et al. 2004. Phase 1 clinical trial of oral curcumin: Biomarkers of systemic activity and compliance. Clin Cancer Res, 10:6847-54. 